Universidad Católica del Norte

Antofagasta - Chile

\title{
GRAFOS CON GRUPO DADO DE AUTOMORFISMOS
}

\author{
Héctor Hevia * \\ Universidad Católica de Valparaíso, Valparaíso
}

\begin{abstract}
Resumen
En un famoso articulo publicado en 1938, Roberto Frucht probó que si $\Gamma$ es un grupo finito dado, entonces existe un grafo cuyo grupo de automorfismos es isomorfo a $\Gamma$. La publicación de Frucht de 1938 fue seguida por otra en 1949 que, en algún sentido, refinaba su primer resultado. Ambas publicaciones generaron un notable número de articulos sobre temas relacionados, asi como una interesante búsqueda de los grafos más pequeños con grupo de automorfismos isomorfo a un cierto grupo dado $\Gamma$. El propósito de este artículo es hacer una recopilación de las investigaciones realizadas principalmente en esta última dirección.
\end{abstract}

Dedicado a Roberto Frucht con ocasión de su cumpleaños 89 


\section{Introducción}

En el año 1736, Leonhard Euler [12] publicó su solución al conocido problema de los puentes de Königsberg. Tal artículo es considerado como uno de los precursores de la teoría de grafos. (Ver una traducción en [7].) Doscientos años más tarde, en 1936, se publicó el primer libro sobre teoría de grafos. Este libro, escrito por Dénes König [31], marca el inicio de una prolífica etapa de esta teoría.

En su libro, König propuso varios problemas relativos a grafos cuyas soluciones eran desconocidas en ese entonces. En particular, en la página 5 de su libro, König preguntó:

"Cuándo es posible realizar un grupo abstracto dado como el grupo de automorfismos de un grafo y - si éste es el caso - cómo puede construirse tal grafo"?

En un famoso artículo publicado en 1938, Roberto Frucht [14] dio respuesta a esta pregunta probando que si $\Gamma$ es un grupo finito dado, entonces existe un grafo cuyo grupo de automorfismos es isomorfo a $\Gamma$. La solución dada por Frucht es constructiva y consiste en modificar inteligentemente un diagrama de Cayley de $\Gamma$.

La publicación de Frucht de 1938 fue seguida por otra en 1949 (ver [16]) que, en algún sentido, refinaba su primer resultado. Ambas publicaciones generaron un notable número de artículos sobre temas relacionados, así como una interesante búsqueda de los grafos más pequeños con grupo de automorfismos isomorfo a un cierto grupo dado $\Gamma$. El propósito de este artículo es revisar las investigaciones realizadas en torno a esta búsqueda.

\section{Definiciones básicas}

La terminología de la teoría de grafos está tomada de [10]. El grupo de automorfismos de un grafo $G$ se denota por $A u t(G)$. Se dice que un grafo es asimétrico si su grupo de automorfismos es trivial. Por ejemplo, el grafo que consiste de un sólo vértice (usualmente denotado por $K_{1}$ ) es un grafo asimétrico. Decimos que un grafo $G$ representa al grupo $\Gamma$ si $A u t(G) \cong \Gamma$. Recordemos que si un grafo $G$ es no conexo, entonces su complemento $\bar{G}$ es conexo. Además, $A u t(G)=A u t(\vec{G})$. En consecuencia, si existe un grafo con $p$ vértices que representa a un grupo $\Gamma$ dado, entonces también existe un grafo conexo con $p$ vértices que representa a $\Gamma$. 
Sea $\Gamma$ un grupo finito no trivial cuya identidad es $e$. Supongamos que $\Delta=\left\{h_{1}, h_{2}, \ldots, h_{d}\right\}$ es un conjunto de elementos de $\Gamma$ tal que $h_{i} \neq e$, para $i=1,2, \ldots, d$. Se dice que $\Gamma$ está generado por los elementos $h_{1}, h_{2}, \ldots, h_{d}$ si cada elemento de $\Gamma$ puede expresarse como un producto de elementos de $\Delta$. En este caso, los elementos de $\Delta$ se llaman generadores y se dice que $\Delta$ es un conjunto generador de $\Gamma$.

Es claro que todo grupo finito no trivial posee un conjunto generador. A partir de un grupo finito no trivial $\Gamma$ y de un conjunto generador $\Delta$ de $\Gamma$, podemos construir un digrafo $C_{\Delta}(\Gamma)$ llamado el diagrama de Cayley de $\Gamma$ con respecto a $\Delta$. En lo que sigue explicamos su construcción.

El conjunto de vértices de $C_{\Delta}(\Gamma)$ es el conjunto de los elementos del grupo $\Gamma$. Sean $g_{1}, g_{2}$ dos elementos de $\Gamma$. Entonces, $\left(g_{1}, g_{2}\right)$ es un arco de $C_{\Delta}(\Gamma)$ si y sólo si $g_{2}=g_{1} h_{i}$, para algún $h_{i} \in \Delta$. Asociando a cada generador un color diferente, podemos también decir que el arco $\left(g_{1}, g_{2}\right)$ tiene $\operatorname{color} h_{i}$ en el diagrama $C_{\Delta}(\Gamma)$.

Por ejemplo, si $\Gamma$ es el grupo de todas las permutaciones sobre el conjunto $\{1,2,3\}$ y $\Delta=\{a, b\}$, donde $a=(123), b=(12)$, entonces el diagrama de Cayley $C_{\Delta}(\Gamma)$ es el que se muestra en la Figura 1. (Recordar que en este grupo $b a=a^{2} b$.)

Un automorfismo de un diagrama de Cayley $D$ es una permutación de los vértices de $D$ que preserva los arcos, su dirección, y su color. El grupo de los automorfismos de un diagrama de Cayley $C_{\Delta}(\Gamma)$ es denotado por $\operatorname{Aut}\left(C_{\Delta}(\Gamma)\right)$. La siguiente propiedad del grupo de automorfismos de un diagrama de Cayley fue observada por Frucht.

\section{Teorema}

Sea $\Gamma$ un grupo finito no trivial y sea $\Delta$ un conjunto generador de $\Gamma$. Entonces :

$$
\operatorname{Aut}\left(C_{\Delta}(\Gamma)\right) \cong \Gamma
$$

Para mayores detalles relativos a esta demostración y a diagramas de Cayley en general, ver [10]. 
$\mathrm{C}_{\Delta}(\Gamma)$

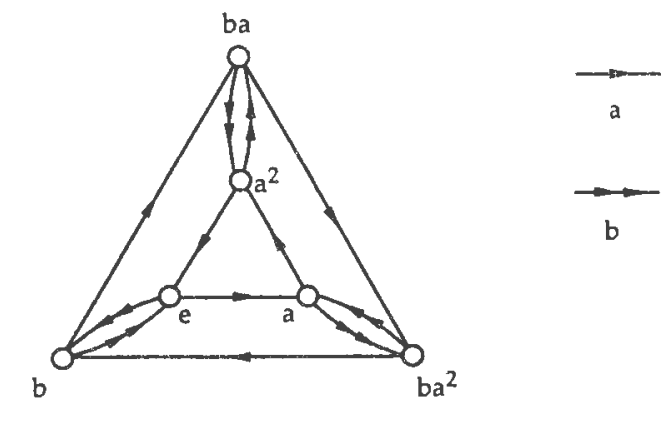

Figura 1 : Un diagrama de Cayley para el grupo simétrico sobre tres símbolos.

\section{El Teorema de Frucht}

Como se mencionó en la introducción, en 1936, König [31] había propuesto determinar cuales grupos (finitos) eran grupos de grafos. A esa sazón, Roberto Frucht se desempeaba como actuario en una compañía de seguros Italiana en Trieste, después de haber obtenido su doctorado en la Universidad de Berlín en el año 1930. (Cabe aquí mencionar que Roberto Frucht había escrito su tesis doctoral en teoría de grupos (ver [13]) bajo la guía de I. Schur, distinguido discípulo de Frobenius. El tema de la disertación de Frucht fue la representación de grupos abelianos finitos mediante colineaciones).

Tal como Frucht lo narra en [19]:

“... un día en 1936 recibí de Akademische Verlagsgesellschaft un catálogo conteniendo una descripción del libro de König. Dado que el Capítulo 8 prometía aplicaciones a teoría de grupos, inmediatamente ordené este libro, y comenzando con el día en que lo recibí yo llegué a ser un entusiasta de la teoría de grafos. Más que en estas "aplicaciones" (las que eran una descripción de los clásicos diagramas de Cayley), yo me interesé en dos problemas relacionados con los grupos de automorfismos de grafos que König formula en su libro." 
Efectivamente, Frucht resolvió ambos problemas formulados por König. En particular, él pudo probar que todo grupo finito es isomorfo al grupo de automorfismos de algún grafo.

\section{Teorema (de Frucht)}

Para cada grupo finito $\Gamma$, existe un grafo $G$ tal que

$$
A u t(G) \cong \Gamma
$$

Con respecto a la demostración de este teorema, el mismo Frucht comenta en [19]:

"Sólo después de varios meses de infructuosos esfuerzos fui suficientemente afortunado como para encontrar una solución la cual parece fácil una vez encontrada: uno tiene sólo que comenzar con el ya m uncionado diagrama de Cayley para el grupo dado. Es verdad que éste no es un grafo ordinario dado que sus líneas están dirigidas y "coloreadas", pero (este diagrama) puede ser transformado en un grafo ordinario - sin modificar el grupo de automorfismos - mediante un truco ..."

La técnica introducida por Frucht para modificar los diagramas de Cayley consiste en reemplazar cada arco coloreado por un grafo (aquí llamado "artefacto") que mantenga las propiedades intrínsecas del color y la dirección del arco. A continuación, ejemplificamos esta técnica construyendo un grafo $G$ que represente al grupo $C_{3}$, el grupo de orden 3 .

Denotemos por $g$ a un generador de $\Gamma \cong C_{3}$. Sea $\Delta=\{g\}$. El diagrama de Cayley $C_{\Delta}(\Gamma)$ se muestra en Figura 2(i). Cada uno de los arcos de $C_{\Delta}(\Gamma)$ se reemplaza por una copia del grafo (artefacto) de la Figura 2 (ii) con el fin de obtener un grafo cuyo grupo de automorfismos es isomorfo a $C_{3}$. El grafo resultante $G$ se muestra en la Figura 2 (iii).

Si $\Delta$ tiene $d$ generadores, es posible reemplazar cada generador $g_{k}, 1 \leq$ $k \leq d$, por un artefacto que agrega $3+2 k$ vértices, cada vez que dicho artefacto sustituye a un respectivo arco coloreado del diagrama de Cayley. De este modo, no es difícil ver que el grafo $G$ construido para probar el teorema de Frucht tiene orden $n(d+1)(2 d+1)$, en donde $n$ es el orden del grupo $\Gamma$. Como $d \leq n-1$, se tiene que $G$ a lo más posee $n^{2}(2 n-1)$ vértices. 
En su artículo del año 1949, Frucht [16] comentaba que este número de vértices era, en algún sentido, excesivo. Además, agregaba lo siguiente:

“... el principal objetivo de este trabajo es dar una nueva solución al problema de König mediante un grafo con menor número de vértices y satisfaciendo la condición adicional de ser regular de grado $3 "$.

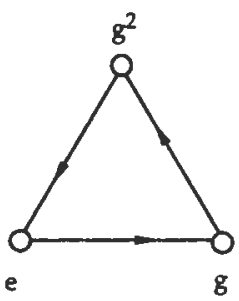

(i)

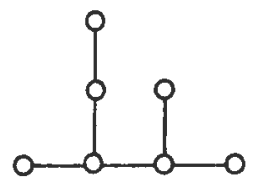

(ii)

G:

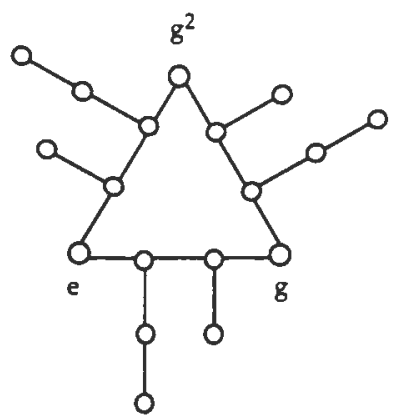

(iii)

Figura 2 : Un ejemplo de la construcción de un grafo 3-cíclico utilizando la técnica de Frucht.

Efectivamente, Frucht logró reducir la cota superior obtenida antes por él mismo. Otros mejoramientos de esta cota fueron obtenidos posteriormente por G. Sabidussi [38] y L. Babai [3]. Denotemos por $v(\Gamma)$ al número mínimo de vértices que puede tener un grafo que represente al grupo $\Gamma$. A continuación, resumimos estos resultados respecto al orden de $v(\Gamma)$. 


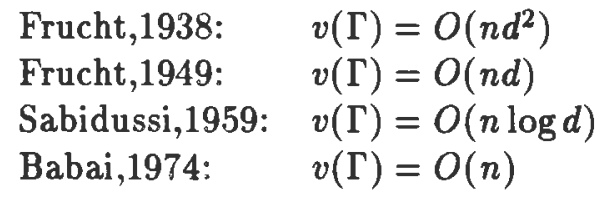

El resultado de Babai es digno de ser mencionado aparte.

\section{Teorema}

Sea $\Gamma$ un grupo de orden $n$. Entonces, $v(\Gamma) \leq 2 n$, excepto si $\Gamma$ es un grupo cíclico de orden 3, 4, ó 5 .

\section{Los valores exactos de $v(\Gamma)$, para ciertos grupos $\Gamma$}

\subsection{El caso del grupo trivial}

La definición de grafo dada por König [31] excluía al grafo $K_{1}$ (lo que no ocurre en la actualidad). Así, para probar su teorema en el caso $|\Gamma|=1$, Frucht presentó el grafo asimétrico $T \not K_{1}$ que se muestra en la Figura 3. Años después, Frucht [19] observó lo siguiente respecto a su publicación de 1938.

“... Curiosamente, yo no me dí cuenta que el mismo truco también trabajaba para el grupo de orden 1 , y así perdí la oportunidad de hallar el grafo asimétrico (no trivial) más pequeño con 6 vértices..."

Este grafo (ver Figura 4), el más pequeño en orden y tamaño que es asimétrico y no isomorfo a $K_{1}$ fue encontrado por I. N. Kagno [29] en 1947.

Es un simple ejercicio probar que si existe un grafo de orden $p>1$ que representa al grupo $\Gamma$, entonces existe un grafo (conexo) de orden $p+1$ que también representa a $\Gamma$. Así, hay grafos asimétricos para todos los órdenes $p \geq 6$. Sin embargo, el tamaño $q$ de estos grafos no puede ser arbitrario. Sea $p \geq 6$ un entero dado. Louis V. Quintas en su tesis doctoral (ver [35]) encontró los valores extremos de $q$ para los cuales existe un grafo asimétrico que tiene $p$ vértices y $q$ aristas. 


\subsection{Los grupos cíclicos $C_{m}, m \geq 2$}

Decimos que un grafo $G$ es $m$-cíclico si $G$ representa al grupo cíclico $C_{m}$ de orden $m \geq 2$. Para simplificar, abreviamos $v\left(C_{m}\right)$ escribiendo $v(m)$. Notemos que el grafo que consiste de dos vértices y una arista $\left(\cong K_{2}\right.$ ) representa al grupo $C_{2}$. Así, $v(2)=2$. Sea $m \geq 3$. En [14], Frucht obtuvo la siguiente cota para $v(m)$ :

$$
v(m) \leq 6 m \text {. }
$$

Más tarde, en 1949, el mismo Frucht [16] mejoró esta cota superior como sigue:

$$
v(m) \leq \begin{cases}10 & \text { si } m=3 \\ 3 m & \text { si } m>3\end{cases}
$$

T:

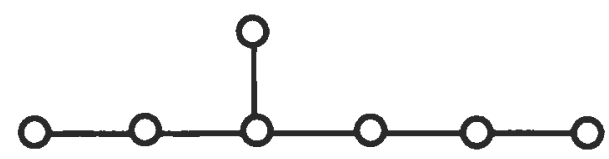

Figura 3 : El árbol asimétrico (no trivial) más pequeño.

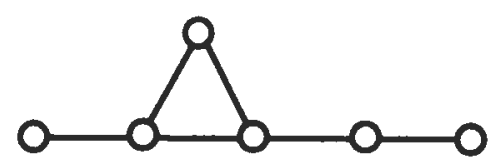

Figura 4 : El grafo asimétrico (no trivial) más pequeño. 
En la Figura 5 mostramos el grafo 3-cíclico de orden 10 utilizado por Frucht para probar que $v(3) \leq 10$. (Esto ilustra el procedimiento general para encontrar una cota superior $B$ de $v(\Gamma)$, el cual consiste en construir un grafo de orden $B$ que representa a $\Gamma$. Así se obtiene $v(\Gamma) \leq B$.)

Después de dar una demostración de la existencia de la cota superior (1), Frucht comenta lo siguiente:

"... quisiéramos enfatizar que podrían haber grafos con todavía menos de $3 \mathrm{~m}$ vértices (a lo menos, para ciertos valores de $m>$ 3 ). Esto se verifica prontamente cuando el orden del grupo cíclico no es un primo ó una potencia de primo. Por ejemplo, sea $m=p_{1}^{e_{1}} p_{2}^{e_{2}}$ ( $p_{1}$ y $p_{2}$ primos distintos); dado que $C_{m}$ es el producto directo de un grupo cíclico de orden $p_{1}^{e_{1}}$ y de un grupo cíclico de orden $p_{2}^{e_{2}}$, y dado que estos grupos tienen grafos con $3 p_{1}^{e_{1}}$ y $3 p_{2}^{e_{2}}$ vértices, estos dos grafos juntos forman un grafo (no conexo) con sólo $3\left(p_{1}^{e_{1}}+p_{2}^{e_{2}}\right)$ vértices ..."

En efecto, supongamos que $G_{1}=\left(V_{1}, E_{1}\right), G_{2}=\left(V_{2}, E_{2}\right)$ son dos grafos conexos no isomorfos. Entonces, el grupo de automorfismos de la unión de los grafos $G_{1}$ y $G_{2}$ está determinado por los grupos de $G_{1}$ y de $G_{2}$. Para precisar, aceptemos que $V_{1} \cap V_{2}=\phi$. Entonces la unión de $G_{1}$ y $G_{2}$, denotada por $G_{1} \cup G_{2}$, es el grafo $\left(V_{1} \cup V_{2}, E_{1} \cup E_{2}\right)$. Bajo estas condiciones,

$$
\operatorname{Aut}\left(G_{1} \cup G_{2}\right) \cong \operatorname{Aut}\left(G_{1}\right) \times \operatorname{Aut}\left(G_{2}\right) .
$$

Quizás, el último comentario citado fue de alguna utilidad para Sabidussi cuando diez años más tarde, en el año 1959, publicó una "solución" al problema de la determinación de $v(m), m \geq 3$. Este resultado (ver[38]) es sólo parcialmente correcto y debería - mejor - entenderse como la determinación de una cota superior para $v(m)$, la que se conjetura como el valor exacto de este parámetro. La cota superior establecida por Sabidussi es la siguiente: 


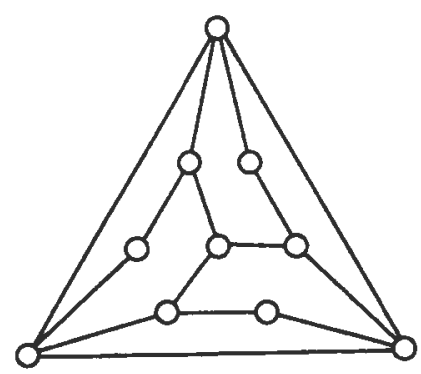

Figura 5 : Un grafo 3-cíclico.

$$
v(m) \leq \begin{cases}2 & \text { si } m=2 \\ 3 m & \text { si } m=3,4,5 \\ 2 m & \text { si } m=p^{e} \geq 7, p \text { primo } \\ \sum_{i=1}^{r} v\left(p_{i}^{e_{i}}\right) & \text { si } m=p_{1}^{e_{1}} \ldots p_{r}^{e_{r}} ; p_{1} \ldots p_{r} \text { primos diferentes }\end{cases}
$$

Los valores exactos de $v(m)$ fueron obtenidos por Robert Meriwether [33] en 1963. Sorprendentemente, este trabajo nunca fue publicado aunque - podría deducirse de su lectura - habría sido presentado para su publicación en Monatshefte für Math.

Pareciera que Frank Harary y Ed Palmer no conocían el trabajo de Meriwether cuando, en 1966, publicaron un artículo [24] en el cual mostraban, para $m=3,4, \mathrm{y} 5$, tres grafos m-cíclicos con $3 m$ vértices pero sólo con $5 \mathrm{~m}$ aristas. (Los correspondientes grafos presentados por Sabidussi tienen $3 m$ vértices y $6 \mathrm{~m}$ aristas, así como los presentados por Frucht en 1949 tienen $3 m$ vértices y $\frac{m^{2}+7 m}{2}$ aristas). De este modo, Harary y Palmer exhibieron - por primera vez en una publicación - dos grafos m-cíclicos (casos $m=3$ y 5 ) que son los más pequeños con respecto al orden y al tamaño. En Figura 6 mostramos el grafo 3-cíclico y el grafo 5-cíclico de Harary y Palmer (denotados aquí por $M_{3}$ y $M_{5}$, respectivamente). Considerando lo breve del artículo de estos autores, todavía queda un par de comentarios que agregar respecto a él. 
$\mathbf{M}_{3}$ :

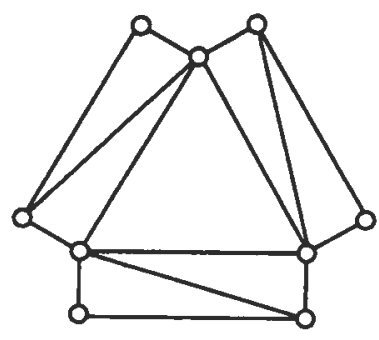

$\mathbf{M}_{5}$ :

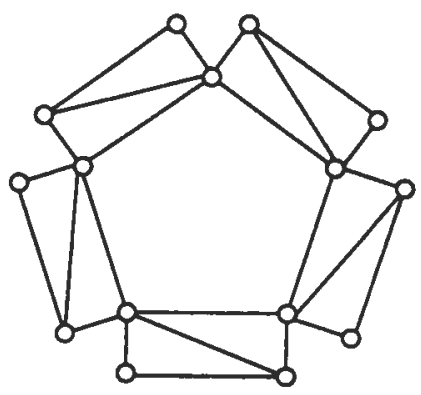

Figura 6 : El grafo 3-cíclico más pequeño y uno de los grafos 5-cíclicos más pequeños.

Pese a que en [24] se afirma que hay exactamente dos grafos no isomorfos más pequeños respecto a orden y tamaño que son 3-cíclicos, el grafo $M_{3}$ de Figura 6 es el único grafo que tiene esta propiedad (ver [8]).

Otro comentario pertinente es que el grafo de Harary y Palmer para $m=4$ no es uno de los grafos más pequeños con respecto al orden (ver fórmulas de Meriwether, más abajo) ni tampoco con respecto al tamaño, como también Bouwer y Frucht lo señalaron en [8].

Todavía el grafo $M_{3}$ guarda otra sorpresa. Cito aquí a Frucht, Gewirtz y Quintas [20].

"Pareciera que ese grafo $\left(M_{3}\right)$ ha sido descubierto en 1956 por Lorraine Goldschmidt (ver [22]); volvió a ser descubierto por Meriwether (1963) quien ha sido el primero en demostrar la propiedad mínima de ese grafo con respecto al número de vértices ... El mismo grafo ha sido descubierto, una vez más por Harary y Palmer (1966) quienes han sido los primeros en publicar dicho grafo, mencionando también su propiedad mínima con respecto al número de aristas".

A continuación, destacamos el resultado de Meriwether. 
Teorcma (de Meriwether)

sen $\rho \geq$ un primo y sea $\epsilon \geq 1$. Entonces:

$$
\begin{aligned}
& v(2)=2 \\
& v\left(2^{e}\right)=2^{e}+6, e \geq 2 \\
& v\left(3^{e}\right)=3^{e}+6 \\
& v\left(5^{e}\right)=5^{e}+10 \\
& v\left(p^{e}\right)=p^{e}+p
\end{aligned}
$$

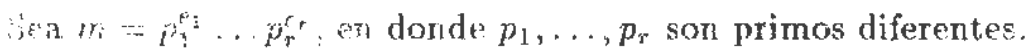

1. Si 3 divide a $m, 25$ no divide a $m$, y si 4 divide a $m$ implica que 8 divide a $m$, entonces

$$
v(m)=\left(\sum_{i=1}^{\tau} v\left(p_{i}^{e_{i}}\right)\right)-3
$$

II. Si 12 divide a $m, 8$ no divide a $m$, y si 5 divide a $m$ implica que 25 divide a $m$, entonces

$$
v(m)=\left(\sum_{i=1}^{r} v\left(p_{i}^{e_{i}}\right)\right)-1
$$

III. Si 60 divide a $m, 8$ no divide a $m$, y si 25 no divide a $m$, entonces

$$
v(m)=\left(\sum_{i=1}^{r} v\left(p_{i}^{e_{i}}\right)\right)^{-4}
$$

IV. Si 24 divide a $m, 9$ no divide a $m$, y si 5 divide a $m$ implica que 25 divide a $m$, entonces

$$
v(m)=\left(\sum_{i=1}^{\tau} v\left(p_{i}^{e_{i}}\right)\right)-1
$$

En todo otro caso,

$$
v(m)=\sum_{i=1}^{r} v\left(p_{i}^{\epsilon_{i}}\right)
$$


En la Figura 7, mostramos el grafo 4-cíclico encontrado por Meriwether en [33]. Aunque este grafo tiene el orden mínimo posible para representar al grupo cíclico de orden 4, no satisface la propiedad de minimalidad con respecto al tamaño (como se probó en [8]).

Una demostración completa del teorema de Meriwether puede encontrarse en [25]. (En [26] está la demostración para el caso en que $m$ es una potencia de primo.) En esta demostración se usa extensamente la denominada representación orbital de grafos que Frucht introdujo en [17] y que fue generalizada en [25].

\subsection{Grupos abelianos (finitos)}

La determinación de $v(\Gamma)$ cuando $\Gamma$ es un grupo abeliano de orden finito fue llevada a cabo por Williams Arlinghaus [1] en 1979. Como era de esperarse (Meriwether lo mencionaba en [33]), un resultado importante en la determinación de los valores de $v(\Gamma)$ es el clásico Teorema Fundamental de los Grupos Abelianos. Este trabajo de Arlinghaus constituyó su tesis doctoral. Se podría ponderar la magnitud e importancia de su disertación, mencionando que un resumen de 85 páginas fue publicado en Memoirs of the American Mathematical Society. (Ver [2].)

\section{Grafos 3-regulares con grupodado}

Un grafo $G$ es regular de grado $k \geq 0$ (k-regular) si el grado de cada vértice de $G$ es $k$. Es inmediato ver que si un grafo es regular de grado impar entonces su orden debe ser par.

En [15] Frucht describió el grupo de un grafo que consiste de $n$ copias de algún otro grafo conexo $G$. (La determinación de este grupo fue el segundo de los problemas planteados por König y que fueron resueltos por Frucht.) El orden de este grupo es $n !|A u t(G)|^{n}$. Recordemos que el grupo de la unión de dos grafos no isomorfos es isomorfo al producto directo de los grupos de estos grafos. Por otro lado, sea $G$ un grafo conexo. Si $G$ es regular de grado 1, entonces $G$ es isomorfo a $K_{2}$; si $G$ es regular de grado 2, entonces $G$ es isomorfo a un ciclo. Estos resultados permiten concluir que hay grupos finitos que no pueden ser representados por grafos regulares de grado $k$, para $k=1,2$. (Considere, por ejemplo, el grupo trivial ó un grupo de orden primo $p \geq 3$.) 


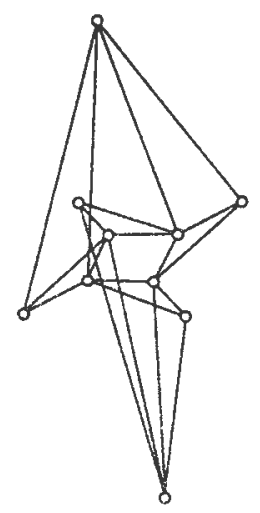

Figura 7 : Uno de los grafos 4-cíclicos de orden mínimo.

Los grafos regulares de grado 3 síque permiten representar a cada grupo $\Gamma$ de orden finito, como así lo demostró Frucht [16] en 1949. Para los casos $|\Gamma|=1$ y $|\Gamma|=2$, Frucht presentó los grafos mostrados en Figuras 8 y 9. En estas figuras, el grafo $F_{1}$ es asimétrico y el grupo del grafo $F_{2}$ tiene orden 2. En el caso general, Frucht es‘ableció ei siguiente resultado.

\section{Teorema}

Si $\Gamma$ es un grupo finito de orden $n \geq 3$ que puede ser generado por $d$ de sus elementos, entonces siempre es posible encontrar un grafo $G$ regular de grado 3 con $2(d+2) n$ vértices que representa a $\Gamma$.

Como el mismo Frucht lo señala en [18] y [19], el número de vértices del grafo $G$ dado en el teorema anterior no es correcto (a lo menos, si uno acepta generadores redundantes para $\Gamma$ ). Al respecto, Babai [4] comentó:

“... El artículo de Frucht del año 1949 tuvo un gran impacto; su mérito no fue solamente probar un teorema sino dar una nueva visión comprensiva sobre el tema - un logro que es alcanzado por pocos artículos sin error alguno."

El mismo Babai, basándose en la construcción original dada por Frucht en [14], dio una demostración del teorema anterior construyendo un grafo regular de grado 3 con $3 n d^{2}+13 n d$ vértices que representa al grupo $\Gamma$ dado. (Ver [32]). Posteriormente, en [4], Babai obtuvo un mejoramiento de este resultado al probar el siguiente teorema. 
$F_{1}$ :

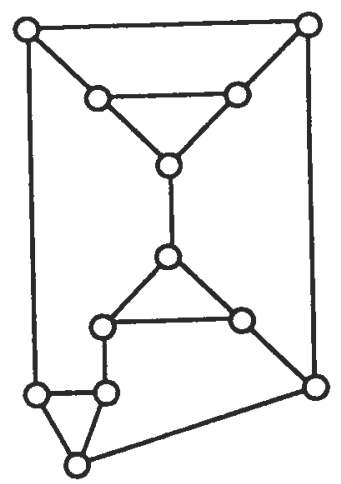

Figura 8 : Un grafo 3-regular asimétrico más pequeño.

$F_{2}$ :

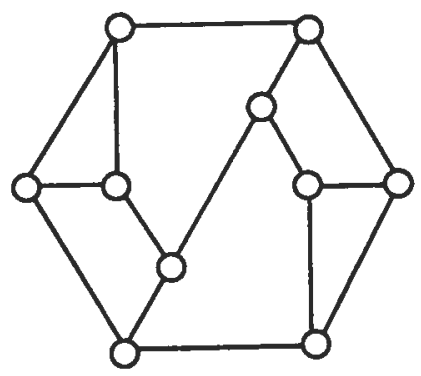

Figura 9 : Un grafo 3-regular 2-cíclico más pequeño.

\section{Teorema}

Si $\Gamma$ es un grupo finito de orden $n \geq 2$ que puede ser generado por $d$ de sus elementos, entonces siempre es posible encontrar un grafo $G$ regular de grado 3 que representa a $\Gamma$ y con a lo más $10 n d+8 n$ vértices.

Denotemos por $v^{3}(\Gamma)$ al número de vértices que puede tener un grafo 3 -regular que represente al grupo finito $\Gamma$. Del teorema anterior se tiene que $v^{3}(\Gamma)=O(n d)$, como Frucht había anticipado. La eficiencia de este resultado queda en evidencia al compararlo con el siguiente teorema de Babai [4]. 


\section{Teorema}

Sea $\Gamma$ un grupo de orden impar $n$ generado por $d$ de sus elementos. Si el grupo $\Gamma$ no puede descomponerse con respecto al producto directo, entonces $v^{3}(\Gamma) \geq \frac{2 n}{3}(d-1)$.

\section{Los valores exactos de $v^{3}(\Gamma)$, para ciertos grupos $\Gamma$}

\subsection{El caso del grupo trivial}

Utilizando el listado de los grafos 3-regulares (y sus diagramas) de orden $p \leq 10$ publicado por A. T. Balaban [5] en 1966, A. T. Balaban, R. O Davies, F. Harary, A. Hill y R. Westwick [6] probaron que $v^{3}(\Gamma)=12$, cuando $\Gamma$ es el grupo trivial. En el mismo artículo, pero esta vez utilizando un listado computacional obtenido en 1966 por D. W. Grace [23], ellos concluyeron que hay exactamente dos grafos 3-regulares planares que son asimétricos y de orden mínimo 12. Estos dos grafos fueron los encontrados por Frucht en 1949. E 1974, L. P. Petrenjuk y A. N. Petrenjuk [34] encontraron todos los grafos 3-regulares cuyo orden es a lo más 12 . Sin embargo, pareciera que en este trabajo no se determinó cuales eran los grupos de automorfismos de estos grafos. En el ao 1976, Kokov [30] probó, con auxilio computacional, que hay cinco grafos 3-regulares asimétricos de orden 12. Estos grafos se muestran en la Figura 10 

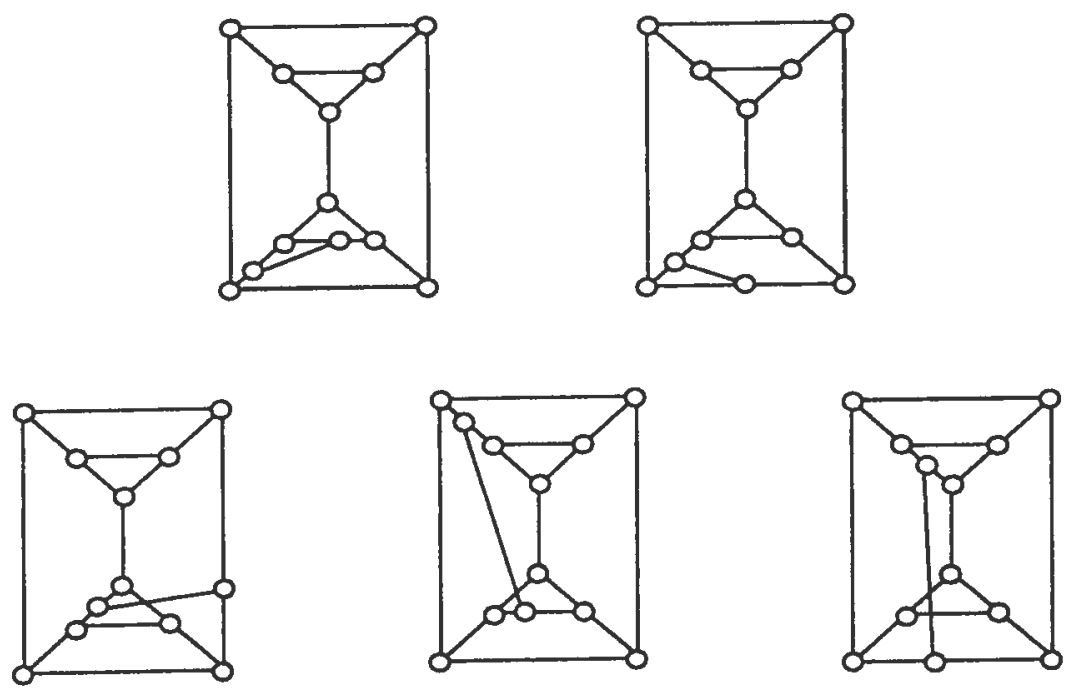

Figura 10 : Los cinco grafos 3-regulares y asimétricos más pequeños.

\subsection{Los grupos cíclicos $C_{m}, m \geq 2$}

En el año 1976, F. C. Bussemaker, S. Cobeljic, D. M. Cvetkovic y J. J. Seidel [9] construyeron un catálogo de grafos 3-regulares de orden menor o igual a 14, calculando, además, ciertos parámetros clásicos de grafos; entre ellos, el orden del grupo de automorfismos. De este trabajo (y también, del listado de diagramas de grafos dado por Balaban en [5], y publicado nuevamente en [6]) puede leerse directamente que sólo hay dos grafos 3regulares más pequeños que representan al grupo $C_{2}$, siendo sólo uno de ellos planar: Precisamente el que Frucht había encontrado en 1949. (En Figura 11 mostramos estos dos grafos.) Así de [9] se obtiene que $v^{3}(2)=10$ (en donde $v^{3}(m)$ es una abreviación para $v^{3}\left(C_{m}\right)$ ). ( ${ }^{*}$ ) 
Del catálogo de Bussemaker et al. también puede concluirse que $v^{3}(3) \geq$ 16. Así, nuevamente Frucht [18] acertó en la búsqueda de los grafos más pequeños, cuando en 1977 mostró el grafo $F_{3}$ de la Figura 12 e hizo el comentario siguiente:

“... (este grafo) es el grafo más pequeño 3-regular con grupo de automorfismos de orden 3 que se conoce."
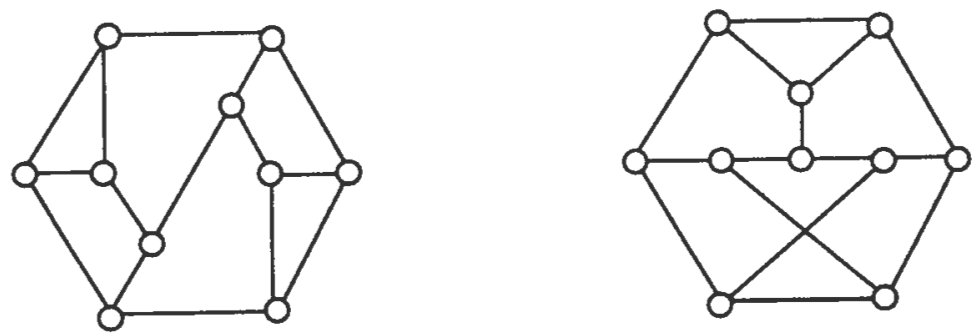

Figura 11 : Los dos grafos 3-regulares 2-cíclicos más pequeños.

$F_{3}$ :
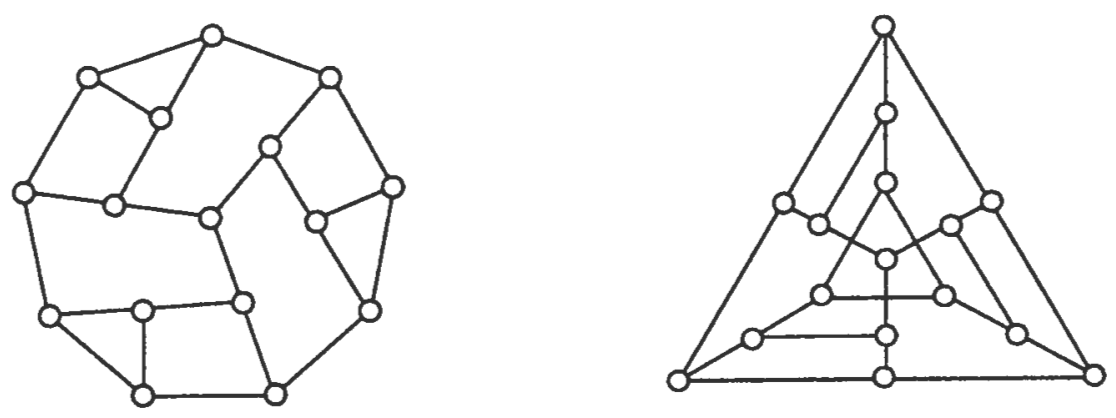

Figura 12 : Los dos grafos 3-regulares 3-cíclicos más pequeños.

De lo anterior, es inmediato que $v^{3}(3)=16$.

El problema de determinar $v^{3}(m)$, para $m \geq 4$, fue resuelto por $\mathrm{H}$. Hevia y S. Ruiz [28] en 1988. En este artículo, sus autores probaron el teorema que sigue. 


\section{Teorema}

$$
v^{3}(m)= \begin{cases}10 & \text { si } m=2 \\ 16 & \text { si } m=3,4 \\ 6 m & \text { si } m=5,7 \\ 2 m+10 & \text { si } 2^{e}, e \geq 3 \\ 4 m & \text { si } m=p^{e} \geq 9, \text { donde p es primo impar }\end{cases}
$$

Sea $m=p_{1}^{e_{1}} \ldots p_{r}^{e_{r}}, r>1$ en donde $p_{1}, \ldots, p_{r}$ son primos diferentes tales que $p_{i}<p_{j}$ siempre que $i<j ; e_{i} \geq 1$ para $i=1,2, \ldots, r$.

I. Si $m=6$, entonces

$$
v^{3}(m)=18
$$

II. Si $m \neq 6$ y $p_{1}^{e_{1}} p_{2}^{e_{2}}=6$, entonces

$$
v^{3}(m)=\left(\sum_{i=3}^{r} v^{3}\left(p_{i}^{e_{i}}\right)\right)+18
$$

En todo otro caso,

$$
v^{3}(m)=\sum_{i=1}^{r} v^{3}\left(p_{i}^{e_{i}}\right)
$$

En la Figura 13 se muestran algunos ejemplos de grafos m-cíclicos más pequeños, para $m=4,5,8$, y 9 . (El grafo 5-cíclico de esta figura, fue encontrado por Frucht en 1949.)

Otros resultados de Hevia y Ruiz en [28] son los siguientes:

(1) Hay exactamente dos grafos 3-regulares más pequeños que representan al grupo de orden 3. (Verlos en Figura 12.)

(2) Existe un único grafo 3-regular más pequeño que representa al grupo cíclico de orden 4. (Este grafo se muestra en Figura 13.) 


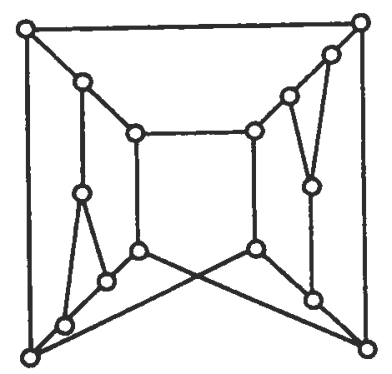

$\mathrm{m}=4$

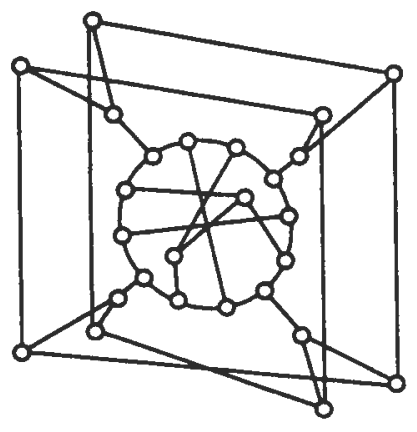

$\mathrm{m}=8$

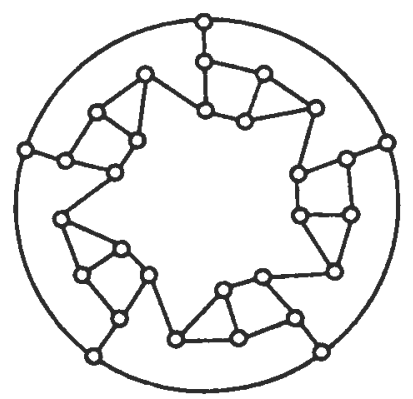

$m=5$

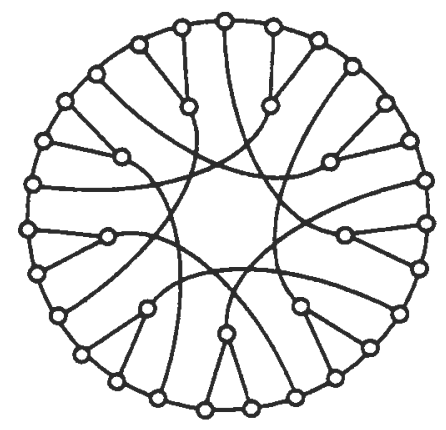

$m=9$

Figura 13 : Algunos grafos 3-regulares m-cíclicos más pequeños.

Sea $m \neq 6$ un entero positivo que no es una potencia de primo. Los grafos 3-regulares más pequeños que representan al grupo cíclico de orden $m$ encontrados por Hevia y Ruiz en [28] no son conexos. Por ejemplo, la unión del grafo 4-cíclico de la Figura 13 y del grafo 5-cíclico de la misma figura es un grafo 3-regular más pequeño en tamaño y orden que representa al grupo cíclico de orden 20. Si queremos representar al grupo cíclico de orden $m$ con un grafo 3-regular y conexo, entonces necesitamos más vértices; como así fue probado en 1991 por Hevia [27].

Denotemos por $v_{c}^{3}(m)$ al número mínimo de vértices que puede tener un grafo conexo 3-regular que represente al grupo cíclico de orden $m$. El siguiente teorema fue demostrado en [27]. 


\section{Teorema}

Sea $m$ un entero positivo y denotemos por $i m p(m)$ al máximo divisor impar de $m$.

Si $m=6$ ó $m=p^{e}, p$ primo.

$$
v_{c}^{3}(m)=v^{3}(m)
$$

Sea $m \geq 10$ un entero que no es una potencia de primo.

I. Si 6 divide a $m$, entonces

$$
v_{c}^{3}(m)=2 m+\frac{10}{3} i m p(m)
$$

II. Si 4 divide a $m$ y si 6 no divide a $m$, entonces

$$
v_{c}^{3}(m)=2 m+4 i m p(m)
$$

En todo otro caso,

$$
v_{c}^{3}(m)=4 m
$$

En la Figura 14 mostramos uno de los grafos más pequeños 3-regular y conexo que represe ta al grupo cíclico de orden 20 .

\section{Grafos k-regulares con grupo dado, $k \geq 4$}

En 1957, Sabidussi [37] probó que dado un grupo finito $\Gamma$ existe un grafo kregular que representa a $\Gamma$, para cada entero $k \geq 4$. De este modo, Sabidussi extendió el resultado de Frucht del año 1949 que establece la universalidad de la clase de los grafos 3-regulares. Este concepto de universalidad fue introducido por Babai en [4]. Una clase de grafos es universal si cada grupo finito puede ser representado por un miembro de la clase. Como ya hemos visto, la clase de los grafos $\mathrm{k}$-regulares, $k=1,2$, no es universal.

\section{Teorema (de Sabidussi)}

Dado un grupo finito $\Gamma$ de orden $n \geq 1$ y un entero $k \geq 3$, existe un número infinito de grafos no isomorfos que son regulares de grado $k$ y cuyo grupo de automorfismos es isomorfo a $\Gamma$. 
La demostración dada por Sabidussi en [37] para probar el teorema anterior es constructiva y se fundamenta en algunos de los grafos definidos por Frucht en [16]. Sabidussi también demostró que la clase de los grafos cuya conectividad es igual a un entero dado $n \geq 1$, es universal. Un resultado similar fue obtenido por Sabidussi para la clase de grafos cuyo número cromático es igual a un entero dado $n \geq 2$.

Denotemos por $v^{k}(\Gamma)$ al número mínimo de vértices que puede tener un grafo k-regular que represente al grupo finito $\Gamma$.

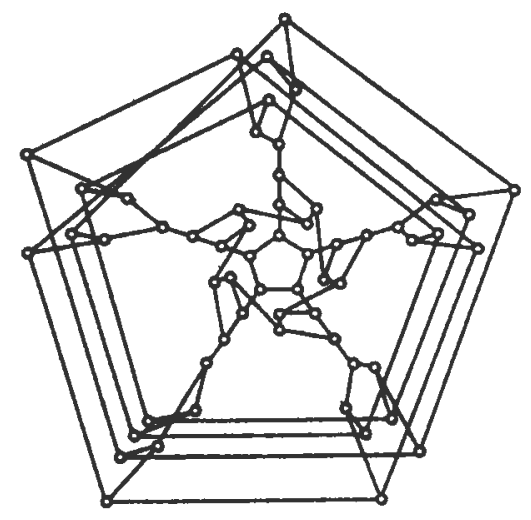

Figura 14 : Un grafo conexo 3-regular 20-cíclico mẩs pequeño.

\section{Los valores exactos de $v^{k}(\Gamma)$, para ciertos grupos $\Gamma, k \geq 4$}

En 1969, A. Gewirtz, A. Hill y L. Quintas [21] determinaron los valores de $v^{k}(\Gamma)$ cuando $\Gamma$ es el grupo trivial y $k \geq 4$. Este artículo, publicado en Español en la revista Scientia, contiene el siguiente teorema como resultado principal.

\section{Teorema}

Sea $\Gamma$ el grupo trivial. Entonces,

(i) $v^{0}(\Gamma)=1$

(ii) No existen grafos asimétricos $\mathrm{k}$-regulares, para $k=1,2$.

(iii) $v^{3}(\Gamma)=12$

(iv) $v^{4}(\Gamma)=v^{5}(\Gamma)=10$

(v) $v^{6}(\Gamma)=11$

(vi) $v^{k}(\Gamma)=k+4, \quad$ si $k \geq 8, k$ par

(vii) $v^{k}(\Gamma)=k+5 \quad$ si $k \geq 7, k$ impar 
En la Figura 15 mostramos el grafo asimétrico 4-regular79dd 0 vértices utilizado por Gewirtz et al. en [21] para probar que $v^{4}(\Gamma) \leq 10$.

En [36], Roland Rambau anunció que todos los grafos 4-regulares de orden 12 ó menor que existen habían sido determinados, obteniendo para cada uno de ellos los valores de ciertos parámetros de grafos; entre ellos, el orden del grupo de automorfismos. Así, de esta clasificación, se obtiene que $v^{4}(\Gamma)=9$ cuando $\Gamma$ es el grupo de orden 2. En la Figura 16 se muestra uno de los tres grafos 4-regulares y 2-cíclicos con 9 vértices que existen. De la clasificación de Rambau también se desprende que hay exactamente 4 grafos asimétricos 4-regulares con 10 vértices.

Recientemente, J. Dávila, R. Giudici y S. Ruiz (ver [11]) determinaron los valores de $v^{4}(\Gamma)$ cuando $\Gamma$ es isomorfo a un grupo cíclico de orden $m \geq 3$. Presentamos este resultado en el siguiente teorema. (A continuación, ano$\operatorname{tamos} v^{4}(m)$ para abreviar $v^{4}\left(C_{m}\right)$.)

\section{Teorema}

$$
v^{4}(m)= \begin{cases}2 m+5 & \text { si } m=2^{e}, e \geq 1 \\ 14 & \text { si } m=3 \\ \frac{8 m}{3} & \text { si } m=3^{e}, e>1 \\ 20 & \text { si } m=5 \\ 3 m & \text { si } m=p^{e} \geq 7, p \geq 5 \text { primo }\end{cases}
$$




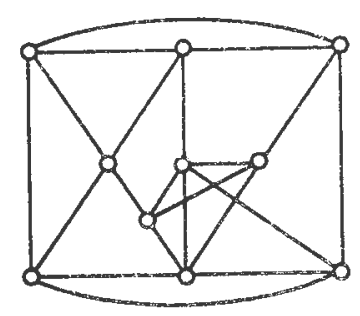

Figura 15 : Uno de los cuatro grafos 4-regulares asimétricos más pequeños.

Sea $m=p_{1}^{e_{1}} \ldots p_{r}^{e_{r}}, r>1$, en donde $p_{1} \ldots, p_{r}$ son primos diferentes; $e_{i} \geq 1$, para $i=1,2, \ldots, r$.

I. $v^{4}(6)=14$

II. $v^{4}(10)=25$

III. En todo otro caso,

$$
v^{4}(m)=\sum_{i=1}^{r} v^{4}\left(p_{i}^{e_{i}}\right)
$$

En la Figura 17 mostramos el grafo 4-regular y 3 cíclico más pequeño encontrado por Dávila, Guidici v Ruiz en [11].

A pesar de lo que uno podría esperar, pareciera que no existe en la literatura resultados sobre el parámetro $v^{k}(\Gamma), k \geq 5$, cuando $\Gamma$ es el grupo de orden 2, ó cuando $\Gamma$ es el grupo de orden 3 . Menos todavía, para el caso más general cuando el orden de $\Gamma$ es un primo. 


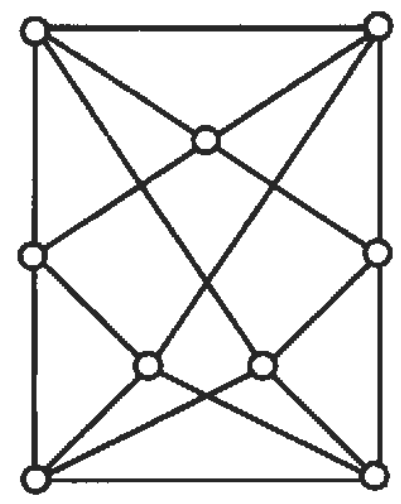

Figura 16 : Uno de los tres grafos 4-regulares 2-cíclicos más pequeños.

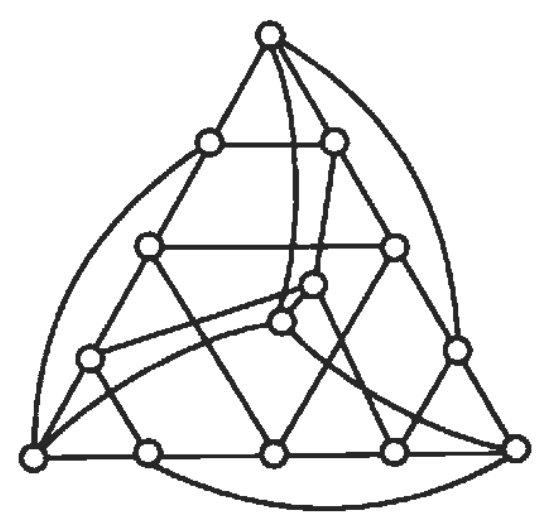

Figura 17 : Un grafo 4-regular 3-cíclico más pequeño. 


\section{Agradecimientos}

El autor desea agradecer la gentil invitación que recibió de Brian Alspach para visitar Simon Fraser University. Durante esa visita, el autor elaboró una versión preliminar de este trabajo.

Muy especiales agradecimientos a Héctor Hevia Jr., quien con mucho esmero realizó algunos de los dibujos que se encuentran en este artículo.

\section{Referencias}

[1] W. Arlinghaus, The structure of minimal graphs with given abelian automorphism group. Ph. D. dissertation, Wayne State University (1979).

[2] W. Arlinghaus, The classification of minimal graphs with given abelian automorphism group. Memoirs Amer. Math. Soc. 57 No. 330 (1985).

[3] L. Babai, On the minimum order of graphs with given group. Canad. Math. Bull. 17, pp. 467-470 (1974).

[4] L. Babaj, On the abstract group of automorphisms. Combinatorics, Cambridge University Press, London, pp. 1-40 (1981).

[5] A. T. Balaban, Valence-isomerism of cyclopolyenes. Rev. Roumaine Chim. 11, pp. $1097-1116$ (1966); erratum: 12, pp. 103 (1967).

[6] A. T. Balaban, R. O. Davies, F. Harary, A. Hill y R. Westwick, Cubic identity graphs and planar graphs derived from trees. Austral. Math. Soc. 11, pp. 207-215 (1970).

[7] N. L. Biggs, E. K. Lloyd y R. J. Wilson, Graph Theory 1736-1936. Oxford University Press, London (1976).

[8] I. Z. Bouwer y R. Frucht, Line-minimal graphs with cyclic group. A Survey of Combinatorial Theory (ed. J. N. Srivastava). North Holland, New York, pp. 53-67 (1973).

[9] F. C. Bussemaker, S. Cobeljic, D. M. Cvetkovic y J. J. Seidel, Computer Investigation of Cubic Graphs, T. H. Report 76-WSK-01 Technological University, Eindhoven, Netherlands (1976).

[10] G. Chartand y I. Iesniak, Graphs and Digraphs, 2nd. Edition. Wadsworth \& Brooks/Cole, Monterey (1986). 
[11] J. Dávila, R. Giudici y S. Ruiz, Smallest tetravalent graphs with a given cyclic group of automorphisms. Notas de la Sociedad de Matemática de Chile X, pp. 101-109 (1991).

[12] L. Euler, Solutio problematis ad geometriam situs pertinentis. Comment. Academiae Sci. I.Petropolitanae 8, pp. 128-140 (1736).

[13] R. Frucht, Über die Darstellung endlicher Abelscher Gruppen durch Kollineationen. J. Reine Angew. Math. 166, pp. 16-29 (1931).

[14] R. Frucht, Herstellung von Graphen mit vorgegebener abstrakter Gruppe. Compositio Math. 6, pp. 239-250 (1938).

[15] R. Frucht, On the groups of repeated graphs. Bull. Amer. Math. Soc. 55, pp. 418-420 (1949).

[16] R. Frucht, Graphs of degree three with a given abstract group. Canad. J. Math 1, pp. 365-378 (1949).

[17] R. Frucht, How to describe a graph. Ann. N. Y. Acad. Sci. 175, pp. 159-167 (1970).

[18] R. Frucht, A canonical representation of trivalent hamiltonian graphs. J. Graph Theory 1, pp. 45-60 (1977).

[19] R. Frucht, How I became interested in graphs and groups. J. Graph Theory 6, pp. 101-104 (1982).

[20] R. Frucht, A. Gewirtz and L. V. Quintas, El número mínimo de líneas para grafos conexos con grupo de automorfismos de orden 3 . Scientia 142, pp. 72-85 (1971).

[21] A. Gewirtz, A. Hill y L. V. Quintas, El número mínimo de puntos para grafos regulares y asimétricos. Scientia 138, pp. 103-111 (1969).

[22] L. Goldschmidt, Linear graphs and groups. Masters' thesis, Brooklyn College, (1956).

[23] D. W. Grace, Computer search for non-isomorphic convex polyhedra. Stanford Computation Center Technical Report CS 15(1956).

[24] F. Harary y E. M. Palmer, The smallest graph whose group is cyclic. Czech. Math. J. 16, pp. 70-71 (1966). 
[25] H. Hevia, Sobre el número mínimo de puntos para que un grafo nciclico exista. Tesis de Magister, Universidad Técnica Federico Santa María (1976).

[26] H. Hevia, Representación orbital de grafos y número mínimo de puntos para grafos n-cíclicos, $n$ una potencia de primo. Scientia 148, pp. 102-122 (1977).

[27] H. Hevia, Smallest connected cubic graphs with a given cyclic group of automorphisms. Graph Theory, Combinatorics, Algorithms and Applications. (eds. Y. Alavi. F. R. K. Chung, R. L. Graham, and D. F. Hsu) SIAM Publications, Philadelphia, pp. 212-229 (1991).

[28] H. Hevia y S. Ruiz, Smallest trivalent graphs with a given cyclic group of automorphisms. Scientia Series A: Math. Sc. 2, pp. 53-73 (1988).

[29] I. N. Kagno, Desargues' and Pappus' graphs and their groups. Amer. J. Math. 69, pp. 859-862 (1947).

[30] V. A. Kohov, All cubic graphs of least order with a trivial automorphism group. Programmirovanie No. 4, pp. 106-107, 112. (1976) (Ver MR 54 \# 12565.)

[31] D. König, Theorie der endlichen und unendlichen Graphen (Kombinatorische Topologie der Streckenkomplexe). Akademische Verlagsgesellschaft, Leipzig (1936).

[32] L. Lovász, Combinatorial Problems and Exercises. North-Holland, New York (1979).

[33] R. L. Meriwether, Smallest graphs with a given cyclic group. (1963) No publicado, ver MR 33 \# 2563.

[34] Petrenjuk L. P. y Petrenjuk A. N., On constructive enumeration of 12 vertex cubic graphs. Combinatorial Analysis 3 Moscow (1974).

[35] L. V. Quintas, Extrema concerning asymmetric graphs. J. Comb. Th. 3, pp. 57-82 (1967).

[36] R. Rambau, Least order 4-regular DDR identity graphs. Notes from New York Graph Theory Day V (eds. J. W. Kennedy and L. V. Quintas) The New York Academy of Sciences, pp. 22-23 (1982). 
[37] G. Sabidussi, Graphs with given group and given graph-theoretical properties. Canad. J. Math. 9, pp. 515-525 (1957).

[38] G. Sabidussi, On the minimum order of graphs with given automorphism group. Monatsh. Math. 63, pp. 124-127 (1959).

Recibido: Agosto 10, 1995.

Héctor Hevia

Instituto de Matemáticas

Universidad Católica de Valparaíso

Casilla 4059

Valparaíso - Chile 